\title{
Inducible responses to antagonistic predation risks are not in a dilemma: Evidences from multi-traits and transcriptome of Ceriodaphnia
}

\author{
Lei $\mathrm{Gu}^{1}$, Shanshan Qin ${ }^{1}$, Yunfei Sun ${ }^{1}$, Jing Huang ${ }^{1}$, Siddiq Akbar ${ }^{1}$, Lu Zhang ${ }^{1}$, and Zhou \\ Yang $^{1}$ \\ ${ }^{1}$ Nanjing Normal University - Xianlin Campus
}

July 7, 2021

\begin{abstract}
Inducible defenses of prey are evolved under diverse and variable predation risks. In the co-evolution of prey and multiple predators with antagonistic selection pressures, whether inducible defense responses of prey will fall into a dilemma and its underlying mechanism are still unclear. Based on the antagonistic predation pressure from invertebrate predator Chaoborus larvae and vertebrate predator fish, we studied multi-traits and transcriptome of the freshwater crustacean Ceriodaphnia cornuta under multiple predation risks. Our results showed that Chaoborus larvae predation risks altered the expression of genes encoding cuticle protein and changed the biosynthesis of steroid hormone, cutin, suberine, and wax, promoting Ceriodaphnia to express horns and grow larger at a late development stage, whereas fish predation risks mainly triggered responses in genes encoding ribosome and pathways of unsaturated fatty acids biosynthesis, cysteine and methionine metabolism, resulting in a smaller individual size and earlier reproduction. The inducible responses on transcription and individual traits both revealed that predator unique responses are dominant and the antagonistic responses are the least. Besides, Pearson correlations between different predator unique responses are extremely weak. Furthermore, the unique individual traits triggered by different predators can be expressed simultaneously. These results indicated that Ceriodaphnia can avoid the dilemma by performing predator unique responses and diverse inducible responses are favored in the co-evolution of zooplankton and multiple predators.
\end{abstract}

Inducible responses to antagonistic predation risks are not in a dilemma: Evidences from multi-traits and transcriptome of Ceriodaphnia

\section{Running title: Inducible responses to multiple predators}

Lei Gu, Shanshan Qin, Yunfei Sun, Jing Huang, Siddiq Akbar, Lu Zhang, Zhou Yang*

Jiangsu Province Key Laboratory for Biodiversity and Biotechnology, School of Biological Sciences, Nanjing Normal University, 1 Wenyuan Road, Nanjing 210023, China

* Corresponding author: Zhou Yang

TEL: +86-25-85891671.

E-mail: yangzhou@njnu.edu.cn

Jiangsu Province Key Laboratory for Biodiversity and Biotechnology, School of Biological Sciences, Nanjing Normal University, 1 Wenyuan Road, Nanjing 210023, China 


\begin{abstract}
Inducible defenses of prey are evolved under diverse and variable predation risks. In the co-evolution of prey and multiple predators with antagonistic selection pressures, whether inducible defense responses of prey will fall into a dilemma and its underlying mechanism are still unclear. Based on the antagonistic predation pressure from invertebrate predator Chaoborus larvae and vertebrate predator fish, we studied multi-traits and transcriptome of the freshwater crustaceanCeriodaphnia cornuta under multiple predation risks. Our results showed that Chaoborus larvae predation risks altered the expression of genes encoding cuticle protein and changed the biosynthesis of steroid hormone, cutin, suberine, and wax, promoting Ceriodaphnia to express horns and grow larger at a late development stage, whereas fish predation risks mainly triggered responses in genes encoding ribosome and pathways of unsaturated fatty acids biosynthesis, cysteine and methionine metabolism, resulting in a smaller individual size and earlier reproduction. The inducible responses on transcription and individual traits both revealed that predator unique responses are dominant and the antagonistic responses are the least. Besides, Pearson correlations between different predator unique responses are extremely weak. Furthermore, the unique individual traits triggered by different predators can be expressed simultaneously. These results indicated that Ceriodaphnia can avoid the dilemma by performing predator unique responses and diverse inducible responses are favored in the co-evolution of zooplankton and multiple predators.
\end{abstract}

Keywords: Cladoceran; Chaoborus ; Fish; Inducible defense; Predation risk

\title{
Introduction
}

In the co-evolution of predator and prey, defense is critical to the survival of prey. Because of the changing predation risks, inducible defenses, triggered by predation cues, are favored by prey (Tollrian \& Harvell, 1999). To successfully defend predators, prey organisms can perform various inducible defensive traits, including behavior (De Meester, 1993), morphology (Gu et al., 2021), chemical substance (Selander et al., 2015), and life history (Kvile, Altin, Thommesen, \& Titelman, 2021). Since diverse predation risks can prevent the stable expression of an inducible defensive trait (Steiner \& Auld, 2012), the present study seeks to understand how a prey response to multiple predation risks, especially to predators with antagonistic selection pressures.

Inducible defenses are common in aquatic organisms, such as phytoplankton (Lürling, 2020), zooplankton (Diel, Kiene, Martin-Creuzburg, \& Laforsch, 2020), amphibians (Mitchell, Bairos-Novak, \& Ferrari, 2017), and fish (Brönmark \& Miner, 1992). Through integration of inducible defense researches, the present study classified the responses against multiple predators into two major categories. The first type is the general response, which evolves through diffusion co-evolution and is a reciprocal adaptation to similar predators, for example, mayfly preforms the same avoidance behavior under different fish predation risks (Alvarez, LandeiraDabarca, \& Peckarsky, 2014); the other type is the specific response, which is evolved by pairwise co-evolution between specific predator and prey, such as the immune responses of immune systems to pathogens (Westra et al., 2015) and the inducible crests of Daphnia in response toNotonecta predation (Grant \& Bayly, 1981). By considering the specific responses in different traits, they can be further subdivided into antagonistic responses on the same trait and unique responses on separate traits. Assuming the selection pressures are antagonistic, if the prey mainly performs antagonistic responses on the same traits, then inducible responses to one predator may cause the prey suffer an environmental cost, i.e., vulnerable to the other predator; if the unique responses on separate traits dominate, then the complex defense responses may create maintenance costs, i.e., energetic costs of the sensory and regulatory mechanisms (Auld, Agrawal, \& Relyea, 2010). Based on this, we cannot theoretically speculate whether inducible responses of prey against antagonistic predation risks are limited and in a dilemma.

In aquatic ecosystems, cladocerans are in the middle of the food chain, providing food resources for insects and fishes (Miner, De Meester, Pfrender, Lampert, \& Hairston, 2012). These invertebrate and vertebrate predators constitute antagonistic selection pressures on the size or habitat selection of waterfleas, for example, larger 
plankton is vulnerable to the large visual predator fish, while less vulnerable to the small ambush predator Chaoborus larvae (Swift, 1992). Thereby, antagonistic inducible traits are commonly expressed byDaphnia , for example, Daphnia hyalina shows completely opposite response in size and reproduction under fish and invertebrates predation pressure (Stibor \& Lüning, 1994); Daphnia galeataprefers deeper habitat under fish predation risk, while inhabits the upper layer under Chaoborus larvae predation risk (Dodson, 1988). Besides, unique inducible defensive traits are prominent inDaphnia, such as "twist" (Herzog, Rabus, Ribeiro, \& Laforsch, 2016), neck teeth (Tollrian, 1993), and horns (Gu, Qin, Zhu, et al., 2020). Furthermore, general responses are noticeable, such as the elongated tail spine, which is observed in Daphnia in response to fish, Triops, and Notonecta (Gu, Qin, Lu, et al., 2020; Ritschar, Rabus, \& Laforsch, 2020). Consistent with the summary of inducible defensive traits, both general and specific responses appear at molecular levels, for instance, Daphnia magna decreases actin and tubulin expression under the predation risk of Chaoboruslarvae or fish (Pijanowska \& Kloc, 2004), increases the expression of ribosomal protein and vitellogenin under fish predation risks (Effertz, Mueller, \& von Elert, 2015), while decreases the expression of vitellogenin and increases cuticle protein under Triops predation risks (Otte, Fröhlich, Arnold, \& Laforsch, 2014). Therefore, in the summary of Daphnia researches, this typical research organism shows various types of responses under antagonistic predation risks. However, through these scattered studies on different Daphniaspecies and clones, we still cannot conclude which type of response is preferred by a prey.

Ceriodaphnia cornuta is a widely distributed species with sensitive inducible defensive traits (Gu et al., 2021; Qin et al., 2021), providing a suitable organism for answering the above question. Since some inducible traits are hidden (Laforsch, Ngwa, Grill, \& Tollrian, 2004), researches on a few traits are not sufficient. In recent years, omics technologies promote our understanding of the mechanisms of inducible defenses (Hales et al., 2017; Zhang et al., 2021). Therefore, to systematically answer how a prey response to antagonistic predation risks, the present study tested multi-traits and transcriptome of C. cornuta in response to Chaoboruslarvae and fish. Besides, to better understand the strategy of inducible responses, we analyzed the Pearson correlation between different inducible responses.

\section{Materials and methods}

\section{Predation risks}

The predation risks were simulated by different predator-conditioned medium, which was prepared according to Gu, Qin, Zhu, et al. (2020). We cultured 4 Rhodeus ocellatus or 100 Chaoborus sp. larvae in aged tap water and fed enough $C$. cornuta for $6 \mathrm{~h}$, and then transferred them into $2 \mathrm{~L}$ of COMBO medium (Kilham, Kreeger, Lynn, Goulden, \& Herrera, 1998) for $18 \mathrm{~h}$. The stock predator-conditioned medium, containing different predator kairomones (Hahn, Effertz, Bigler, \& von Elert, 2019; Weiss et al., 2018), were filtered through a $0.22 \mu \mathrm{m}$ glass fiber filter (Millipore) and then the filtrates were stored in a refrigerator before the experiments. To test the response ofCeriodaphnia against fish, as well as Chaoborus, we set up a full factor experiment containing the following treatments: The control $(\mathrm{C})$ treatment was COMBO medium; Fish $(\mathrm{F})$ and Chaoborus $(\mathrm{CH})$ predation risk treatments were produced by diluting their filtered stock medium 20 times (i.e., 1 fish per $10 \mathrm{~L}$ ) and 2.5 times (i.e., 20 Chaoborus larvae per L) in COMBO medium, respectively; the combination treatment $(\mathrm{CH}+\mathrm{F})$ consisted of the above two diluted medium.

\section{Life history experiment}

The C. cornuta clone used in the present study was sampled from Lake Taihu $\left(31^{\circ} 22^{\prime} 13.548^{\prime \prime} \mathrm{N}\right.$, 120deg0'16" E), China. We cultured C. cornuta in COMBO medium and fed with Chlorella pyrenoidosa $(1.5 \mathrm{mg} \mathrm{C} / \mathrm{L})$ at $25 \mathrm{degC}$ under a fluorescent light intensity $500 \mathrm{Lux}$ in a 14:10 h light/dark cycle. Synchronous C. cornuta with a density of 1 ind per $10 \mathrm{~mL}$ were adapted to the above conditions for at least two generations. We randomly divided newborn individuals into different treatments within $12 \mathrm{~h}$. Each individual was cultured in $10 \mathrm{~mL}$ medium with 10 replicates for each treatment and the media in different treatments were refreshed daily. 
The body size and horns were detected at maturity and a late developmental stage, i.e., the 16th day. We scored the horns of C. cornuta according to Gu et al. (2021), i.e., absent (score 0), small (score 5), and large (score 10), and then the individual scores were normalized by a maximum point to define the induction levels between $0 \%$ and $100 \%$. Besides, time to the first brood, neonate size, brood number, total offspring number, and average brood size were recorded in the present study.

\section{RNA samples and sequencing}

To further analyze the type of responses on the transcriptional level, we sequenced the transcriptome of $C$. cornuta under C, F, and $\mathrm{CH}$ treatments. Groups of 250 newborn individuals were cultured in $2.5 \mathrm{~L}$ medium with 3 replicates for each treatment. During this cultivation, responses triggered by different predation risks were verified through inducible traits, i.e., horns and body size at maturity. We refreshed the medium daily and took samples within $12 \mathrm{~h}$ after the first brood of C. cornuta. Ceriodaphnia samples were frozen in liquid nitrogen and homogenized in TransZol Up, and the total RNA was extracted using TransZol Up Plus RNA Kit following the manufacturer's instructions (ER501, TRANS, China). RNA quality was assessed by an Agilent 2100 Bioanalyzer (Agilent Technologies, USA) and checked by agarose gel electrophoresis. In the present study, the RNA integrity number of all samples was above 7.0.

The total mRNA of Ceriodaphnia was enriched by Oligo (dT) beads, then the enriched mRNA was fragmented into short fragments using fragmentation buffer and reverse transcribed into cDNA with random primers. The cDNA fragments were purified, end repaired, poly(A) added, and ligated to Illumina sequencing adapters, then the ligation products were size selected and PCR amplified to develop a cDNA library. Finally, the cDNA library was sequenced using Illumina HiSeq ${ }^{\mathrm{TM}} 4000$ by Gene Denovo Biotechnology Co. (Guangzhou, China).

\section{Transcript assembly and annotation}

Since the genomic sequencing in Ceriodaphnia has not been conducted to date, we adopted De Novo RNASeq to analyze the transcriptome of $C$. cornuta. To get high-quality clean reads, sequenced reads were cleaned up by removing reads containing adapters, more than $10 \%$ of unknown nucleotides $(\mathrm{N})$, and lowquality reads (Q-value[?]20). Clean reads were assembled into unigenes using the Trinity program (Grabherr et al., 2011). To annotate the unigenes, we used the BLASTx program with an E-value $\left(<10^{-5}\right)$ to NCBI non-redundant protein $(\mathrm{Nr})$ database, the Swiss-Prot protein database, the Kyoto Encyclopedia of Genes and Genomes (KEGG) database, and the COG/KOG database. Protein functional annotations could then be obtained according to the best alignment results.

\section{Quantification of gene expression and RT-qPCR Analysis}

The unigene expression was calculated and normalized to RPKM (Reads Per kb per Million reads) (Mortazavi, Williams, McCue, Schaeffer, \& Wold, 2008). RNAs differential expression analysis was performed by DESeq2 software between control and different predation risk treatments. The genes with the parameter of false discovery rate (FDR) below 0.05 and absolute fold change [?] 1 were considered differentially expressed genes (DEGs). We classified the DEGs into different types according to our classification and then used KEGG analysis to classify the function of DEGs. Pathways with $P$-value [?] 0.05 were considered as significantly enriched pathways. The representative DEGs of different significantly enriched pathways were selected according to the following precedence conditions: stable expression, large fold change, and the pathways are annotated on a closely related species, i.e., D. magna and D. pulex .

To validate the RNA-Seq data in $C$. cornuta transcriptome, we quantitated the expressions of 18 random DEGs by RT-qPCR. ddH2O was used as the negative control in RT-qPCR. The cDNA was synthesized from mRNA by Reverse Transcriptase SuperMix (R233, Vazyme, China), and RT-qPCR was conducted using ChamQ Universal SYBR qPCR Master Mix (Q711, Vazyme, China). All the primer sequences are presented in Supplementary Table 1. We obtained expression data from four alternative reference genes (Scoville \& 
Pfrender, 2010) and calculated their average gene expression stability by geNorm. The glyceraldehyde3-phosphate dehydrogenase (G3PD), the RNA polymerase II gene (RNAP II), and the elongation factor 1-alpha gene $(\mathrm{EF})$ were determined to be stably expressed and were geometrically averaged to calculate a gene expression normalization factor for each sample. Gene expression was calculated using $2^{-\Delta \Delta \tau}$ method. Correlation between RNA-Seq and RT-qPCR was performed by regression analysis.

\section{Statistical Analysis}

To test the effects of different predation risks, a MANOVA, followed by two-way ANOVA, was performed on individual traits. The significant differences $(P<0.05)$ among different treatments were tested by the Bonferroni test. When the normality test (Shapiro-Wilk) failed, we used Scheirer-Ray-Hare followed by Wilcoxon rank-sum test to analyze the differences between treatments. To test the relationship between different types of response, we analyzed the Pearson correlation coefficient between traits, as well as representative DEGs, in different categories. The statistical tests were performed using $\mathrm{R}$ software (version 3.6.2).

\section{Results}

\section{Morphology and life history traits}

Different predation risks significantly triggered various responses in morphology and life history traits (Table 1). Compared with control, the responses induced by fish and Chaoborus larvae predation risks could be classified into the following four categories (Fig. 1): (1) Unique responses to Chaoborus larvae: horn expression (at maturity and 16th day) and total offspring number, i.e., C. cornuta expressed horns (maturity: $P<0.001 ; 16$ th day: $P<0.001)$ and increased offspring number $(P=0.002)$ under Chaoborus larvae predation risk, and the traits were not significantly changed by fish predation risk. (2) Unique responses to fish: time to first brood and neonate size, i.e., the neonate size $(P=0.006)$ and time to first brood $(P<0.001)$ of $C$. cornuta were remarkably decreased under fish predation risk, and these responses were not significant underChaoborus larvae predation risk. (3) General responses: Size at maturity and brood number, i.e., the size (CH vs. $\mathrm{C}: P=0.007$; F vs. $\mathrm{C}: P<0.001)$ and brood number $(\mathrm{CH}$ vs. $\mathrm{C}: P=$ 0.006 ; F vs. C: $P=0.017$ ) were significantly decreased under fish and Chaoborus larvae predation risks. (4) Antagonistic responses: size at 16th day, i.e., C. cornuta increased the size under Chaoborus larvae $(P=$ $0.034)$, but decreased the size under fish predation risks $(P<0.001)$. Additionally, no significant differences were observed in average brood size. Among all those traits, unique responses to predators were dominant, i.e., unique responses $(5)>$ general responses $(2)>$ antagonistic responses (1).

Interactions between fish and Chaoborus larvae predation risks were noticeable in different traits (Table 1). General responses: size at maturity in the combination treatment was smaller than that in control $(P$ $<0.001)$ and had no significant difference with that in fish predation risk treatments; brood number of the combination treatment did not have remarkable differences with that of other treatments. Unique responses: time to first brood $(\mathrm{CH}+\mathrm{F}$ vs. $\mathrm{C}: P=0.027)$ and neonate size $(\mathrm{CH}+\mathrm{F}$ vs. $\mathrm{C}: P=0.003)$ were significantly decreased in the combination treatment and had no significant differences with those in fish predation risk treatment; horn expression in the combination treatment was significant at maturity $(\mathrm{CH}+\mathrm{F}$ vs. C: $P$ $=0.007$ ), while this inducible trait was greatly impaired when compared with that of Chaoborus larvae predation risk treatment (maturity: $P=0.012$; 16th day: $P<0.001$ ). Antagonistic responses: size at 16th day in the combination treatment was significantly smaller than that in Chaoborus larvae predation risk treatment $(P<0.001)$, higher than that in fish predation risk treatment $(P=0.065)$, and not different from that in control $(P=0.764)$.

\section{Overview of assembled transcriptome}

We obtained 78341484, 72493072, and 69930191 clean reads in $\mathrm{C}, \mathrm{CH}$, and F, respectively. A total of 37120 assembled unigenes were assembled through Trinity software and each sample contained more than $66.54 \%$ assembled unigenes (Supplementary Table 2). By comparing with Nr, KEGG, COG, and SwissProt 
databases, a total of 18343 unigenes (49.4\%) were annotated in these public databases (Supplementary Fig. 1). Regarding the species distribution in the $\mathrm{Nr}$ database, C. cornuta had the highest comparison rate with Daphnia magna (26.33\%), followed by D. pulex (2.6\%) (Supplementary Fig. 2A); the unigenes enriched in the KOG database were classified into transcription, ribosomal structure, and gene replication, recombination, and repair (Supplementary Fig. 2B); the annotated GO terms were mainly associated with metabolic process, cellular process, cell part, and binding (Supplementary Fig. 2C).

\section{Differentially expressed genes (DEGs)}

Paired samples within the same treatment had high Pearson correlation coefficients ([?]0.90) and were clustered together in principal component analysis (Fig. 2A and B), which conformed to requirements of biological repetition. Furthermore, the expression patterns showed a significant correlation between RT-qPCR and RNA-Seq (Fig. 2C (a) $R^{2}=0.936$; (b) $R^{2}=0.788$ ), indicating that our analysis on the expression data by RNA-Seq is reliable.

Compared with control, Chaoborus larvae and fish predation risk significantly affected the expression of 1515 and 846 genes, respectively (Fig. 3A). Among them, there were 1399 unique DEGs in CH, 730 unique DEGs in F, 114 general DEGs, and 2 antagonistic DEGs (Fig. 3B). Therefore, unique responses to predators were dominant at the transcriptional level, i.e., unique DEGs (2129) > general DEGs (114) > antagonistic DEGs (2) (Supplementary Table 3).

Considering the DEGs caused by different predators, we further analyzed the differences in the enriched pathways of C. cornuta (Supplementary Table 4) and concluded the main DEGs and pathways related to inducible defensive traits (Table 2). The DEGs of C. cornutaagainst Chaoborus larvae, including cuticle protein, fatty acyl-CoA reductase, and trypsin genes, were mainly enriched in cutin, suberine and wax biosynthesis, protein digestion and absorption, and steroid hormone biosynthesis; the DEGs of C. cornuta against fish, containing ribosomal protein, actin, and short-chain type dehydrogenase genes, were mainly enriched in cysteine and methionine metabolism, ribosome, phototransduction, and biosynthesis of unsaturated fatty acids; the general DEGS, including cysteine proteinase, HSP70, actin, and alpha-tubulin genes, were enriched in the pathways of apoptosis and antigen processing and presentation.

\section{Correlation analysis between different inducible responses}

Both 9 individual traits and 40 representative DEGs (Supplementary Table 5) revealed high Pearson correlations within unique responses to fish or Chaoborus larvae (Fig. 4), such as the response of horns at maturity was significantly correlated with the changes of horns at 16th day $(P=0.05)$ and total offspring number $(P=0.05)$. Besides, both correlation analyses showed weak Pearson correlation coefficients between unique responses to fish and unique responses toChaoborus larvae. While, a few significant correlations appeared on the weak correlation area in the transcriptional expression, such as Unigene0008297, in ribonucleoprotein component protein expression, had significant positive relationships with Unigene0027903 $(P=0.049)$ and Unigene0005204 $(P=0.013)$ in Aspartokinase and Adenosylhomocysteinase expression, respectively (Fig. 4; Supplementary Table 5).

\section{Discussion}

Our experiments clearly showed that $C$. cornuta performed diverse responses under the antagonistic predation risks consisted of fish and Chaoborus larvae. Based on the classification of inducible responses, our results revealed, for the first time, that predator unique responses are dominant, followed by the general responses, and the antagonistic responses are the least. When the antagonistic predation risks coexist, unique individual traits triggered by different predators can be expressed simultaneously, thereby the Pearson correlations between the unique responses to different predators are very weak. According to these results, this study supports the view that prey prefers predator unique responses in the co-evolution of prey and multiple predators, which may cause complex costs and limitations of inducible defenses. 
Horns and larger size are adaptive inducible traits to Chaoboruslarvae predation (Gu et al., 2021; Riessen $\&$ Trevett-Smith, 2009). The larger individual size at a late development stage requires rapid growth and more food intake (Gianuca, Pantel, \& De Meester, 2016), thereby promoting the brood number and total offspring number. The horns are formed by the carapace, which is composed of two layers of dermal cells and covered by chitin, which combines with cuticle protein (Charles, 2010). Therefore, the formation of morphological defensive traits involves a series of changes in the expression of chitin, hormones, and epidermal formation genes at different times (Christjani, Fink, \& Elert, 2016; Miyakawa et al., 2010), as well as the regulation of epidermal cell growth by endocrine hormones (Weiss, Leese, Laforsch, \& Tollrian, 2015). In this study, significant changes in genes and pathways were involved in cuticle protein, cutin, suberine, and wax biosynthesis, and steroid hormone biosynthesis. These genes' expression may promote the synthesis of related substances (Fig. 5) and regulate individual growth (Edgar, 2006). However, growth and development of cladocerans require continuous molting and formation of new carapace, thus, the maintenance of horns needs continuous substance synthesis, which may result in continuous distribution costs (Auld et al., 2010). Besides, Chaoborus predation risks altered the digestion and absorption of $C$. cornuta, such as the trypsin gene, which may affect the digestion and resource allocation strategy (Von Elert et al., 2004).

The smaller size, earlier reproduction, and increased brood number are adaptive responses to fish predation risks, which are similar to the typical responses of other cladocerans under fish predation (Diel et al., 2020). In terms of gene expressions, our results showed that the genes encoding the proteins of actin and ribosomal are down-regulated under fish predation risks. Since actin plays an important role in the structure of the cytoskeleton, the inhibition of actin may result in a smaller cladoceran size. Similar results were observed in the studies ofD. magna inducible defenses (Effertz et al., 2015; Pijanowska \& Kloc, 2004). On the contrary, Schwarzenberger, Courts, and von Elert (2009) revealed an up-regulation of actin genes in D. magna under fish predation risks. Because gene expression is jointly regulated by transcriptional regulators and related proteins (Stibor, 2002), the differential expressions could be observed within 1-2 hours (Effertz \& von Elert, 2014). Ribosomal proteins are responsible for protein assembly and translation, thus, the down-regulation of ribosomal protein may inhibit the synthesis of proteins that are needed for individual growth and development (Zhou, Liao, Liao, Liao, \& Lu, 2015), ultimately affecting the growth of $C$. cornuta . In the enrichment analysis, some DEGs can be enriched in multiple pathways. The significantly enriched phototransduction may change the visual perception ofDaphnia (Mahato et al., 2014), which could be an adaption to behavioral responses, such as habitat selection (Loose \& Dawidowicz, 1994) and escape behavior (Pietrzak, Pijanowska, \& Dawidowicz, 2017). Besides, fish predation can reduce the unsaturated fatty acids of neonates, causing Daphnia to be vulnerable to starvation (Stibor \& Navarra, 2000), therefore the significantly enriched pathway of unsaturated fatty acids may alter the distribution of unsaturated fatty acids. Furthermore, the longevity regulating pathway was significantly enriched under fish predation risks, which may cause an opportunity cost, i.e., the decline of lifespan (Dawidowicz, Predki, \& Pietrzak, 2010).

When facing different predators, C. cornuta showed general responses, such as the expression of cysteine protease, heat shock protein, actin, and tubulin genes. The cDNA sequence of crustacean cysteine protein is closer to that of insect cathepsin L, which regulates the molting cycle and programs cell death during development (Agrawal, Bagchi, \& Bagchi, 2005). Thus, the affected cysteine protease may affect molting and increase brood number in the present study. The up-regulation of heat shock protein is an adaptive response various environmental stresses, including predation risks (Pijanowska \& Kloc, 2004). Because this response is rapid and returns to previous level after long-term treatment (Pauwels, Stoks, \& De Meester, 2005; Pauwels, Stoks, Decaestecker, \& De Meester, 2007), the down-regulation of heat shock protein genes may promote the recovery of heat shock protein in this study. Similarly, general responses are observed in actin and tubulin genes of Daphnia (Pijanowska \& Kloc, 2004), they are involved in the formation of the cytoskeleton and other life activities, while their specific functions still need further researches (Chen et al., 2018).

From the perspective of different responses, prey performs coupling responses to the same predator and extremely weak coupling unique responses to antagonistic predation risks. It is easy to understand that prey can alter resource allocation strategies under single predation risk, resulting in an array of adaptive responses 
(Reede, 1995). For a successful evolution of predator unique responses under multiple predation risks, we mainly considered it from the genotype, selection, and cost. Firstly, the genotypes of cladocerans in ponds or lakes are highly diverse and the inducible traits of different clones are uncoupled (Boersma, Spaak, \& De Meester, 1998; Decaestecker, De Meester, \& Mergeay, 2009; Stoks, Govaert, Pauwels, Jansen, \& De Meester, 2016). Secondly, in the process of predation, multiple predators have diversified selection effects, which contribute to predator unique defensive traits (Herzog \& Laforsch, 2013; Heynen, Bunnefeld, \& Borcherding, 2017). Finally, the environmental costs, such as changing predator regimes, may exceed maintenance costs ( Decaestecker, De Meester, \& Ebert, 2002; Tollrian, 1995; Yin, Laforsch, Lohr, \& Wolinska, 2011), thus, complex unique responses are favored by prey. In our study, this inducible defensive strategy can avoid the dilemma of responses on single traits, improving the survival rate of prey under multiple predation risks. For example, smaller C. cornuta is less likely to be found by fish (O'brien, 1987). At the same time, horn expression makes C. cornuta less vulnerable toChaoborus larvae predation (Gu et al., 2021). While, the co-expression of unique inducible defenses is influenced by development, indicating that there is a trade-off underlying the adaption to multiple predation risks (Riessen \& Gilbert, 2019). Therefore, further studies are still needed to reveal how prey responses to multiple predators, especially in a complex biological and abiotic environment.

\section{Conclusions}

Through the responses on individual traits and transcription, this study revealed inducible responses of C. cornuta againstChaoborus larvae and fish. To cope with such antagonistic predation risks, C. cornuta mainly changed cuticle gene expression and formed horns under Chaoborus larvae predation risk, while altered ribosome genes expression and reduced body size under fish predation risks. Our analysis on those inducible responses revealed for the first time that different predator unique responses are dominant and extremely weak coupling. Contrary to the dilemma of responses on a few inducible defensive traits, this study supports the view that zooplankton prefer predator unique responses and performs the least antagonistic responses in the adaption to antagonistic predation pressures, implying that the potential co-evolutions with multiple predators are mutually shaping the inducible responses of zooplankton.

\section{Acknowledgments}

This study was supported by the Key Project of National Natural Science Foundation of China (31730105) and the Priority Academic Program Development of Jiangsu Higher Education Institutions.

\section{References}

Agrawal, M. K., Bagchi, D., \& Bagchi, S. N. (2005). Cysteine and serine protease-mediated proteolysis in body homogenate of a zooplankter, Moina macrocopa, is inhibited by the toxic cyanobacterium, Microcystis aeruginosa PCC7806. Comparative Biochemistry and Physiology Part B: Biochemistry and Molecular Biology, 141 (1), 33-41. doi:10.1016/j.cbpc.2005.01.002

Alvarez, M., Landeira-Dabarca, A., \& Peckarsky, B. (2014). Origin and specificity of predatory fish cues detected by Baetis larvae(Ephemeroptera; Insecta). Animal Behaviour, 96 , 141-149. doi:10.1016/j.anbehav.2014.07.017

Auld, J. R., Agrawal, A. A., \& Relyea, R. A. (2010). Re-evaluating the costs and limits of adaptive phenotypic plasticity. Proceedings of the Royal Society B: Biological Sciences, 277 (1681), 503. doi:10.1098/rspb.2009.1355

Boersma, M., Spaak, P., \& De Meester, L. (1998). Predator-mediated plasticity in morphology, life History, and behavior of Daphnia : the uncoupling of responses. The American naturalist, 152 (2), 237-248. doi:10.1086/286164 
Brönmark, C., \& Miner, J. G. (1992). Predator-induced phenotypical change in body morphology in crucian carp. Science, 258 (5086), 1348-1350. doi:10.1126/science.258.5086.1348

Charles, J. P. (2010). The regulation of expression of insect cuticle protein genes. Insect Biochemistry and Molecular Biology, 40 (3), 205-213. doi:10.1016/j.ibmb.2009.12.005

Chen, L., Barnett, R. E., Horstmann, M., Bamberger, V., Heberle, L., Krebs, N., . . Weiss, L. C. (2018). Mitotic activity patterns and cytoskeletal changes throughout the progression of diapause developmental program in Daphnia . BMC cell biology, 19 (1), 30. doi:10.1186/s12860-018-0181-0

Christjani, M., Fink, P., \& Elert, E. V. (2016). Phenotypic plasticity in three Daphnia genotypes in response to predator kairomone: evidence for an involvement of chitin deacetylases. Journal of Experimental Biology, 219 , 1697-1704. doi:10.1242/jeb.133504

Dawidowicz, P., Predki, P., \& Pietrzak, B. (2010). Shortened lifespan: another cost of fish-predator avoidance in cladocerans? Hydrobiologia, 643 , 27-32. doi:10.1007/s10750-010-0132-z

De Meester, L. (1993). Genotype, fish-mediated chemical, and phototactic behavior in Daphnia Magna . Ecology, 74 (5), 1467-1474. doi:10.2307/1940075

Decaestecker, E., De Meester, L., \& Ebert, D. (2002). In deep trouble: Habitat selection constrained by multiple enemies in zooplankton.Proceedings of the National Academy of Sciences, 99 (8), 5481-5485. doi:10.1073/pnas.082543099

Decaestecker, E., De Meester, L., \& Mergeay, J. (2009). Cyclical parthenogenesis in Daphnia : Sexual versus asexual reproduction. In I. Schön, K. Martens, \& P. Dijk (Eds.), Lost Sex: The Evolutionary Biology of Parthenogenesis (pp. 295-316). Dordrecht: Springer Netherlands.

Diel, P., Kiene, M., Martin-Creuzburg, D., \& Laforsch, C. (2020). Knowing the enemy: Inducible defences in freshwater zooplankton.Diversity, 12 (4), 147. doi:10.3390/d12040147

Dodson, S. (1988). The ecological role of chemical stimuli for the zooplankton: Predator-avoidance behavior in Daphnia .Limnology and Oceanography, 33 (6part2), 1431-1439. doi:10.4319/lo.1988.33.6part2.1431

Edgar, B. A. (2006). How flies get their size: genetics meets physiology. Nature Reviews Genetics, 7 (12), 907-916. doi:10.1038/nrg1989

Effertz, C., Mueller, S., \& von Elert, E. (2015). Differential Peptide Labeling (iTRAQ) in LC-MS/MS based proteomics in Daphnia reveal mechanisms of an antipredator response. Journal of proteome research, 14 (2), 888-896. doi:10.1021/pr500948a

Effertz, C., \& von Elert, E. (2014). Light intensity controls anti-predator defences in Daphnia: the suppression of life-history changes. Proceedings of the Royal Society B: Biological Sciences, 281 (1782), 20133250. doi:10.1098/rspb.2013.3250

Gianuca, A. T., Pantel, J. H., \& De Meester, L. (2016). Disentangling the effect of body size and phylogenetic distances on zooplankton top-down control of algae. Proceedings of the Royal Society B: Biological Sciences, 283 (1828), 20160487. doi:10.1098/rspb.2016.0487

Grabherr, M. G., Haas, B. J., Yassour, M., Levin, J. Z., Thompson, D. A., Amit, I., . . . Zeng, Q. (2011). Full-length transcriptome assembly from RNA-Seq data without a reference genome. Nature biotechnology, 29 (7), 644-652. doi:10.3410/f.13296969.14657090

Grant, J., \& Bayly, I. (1981). Predator induction of crests in morphs of the Daphnia carinata King complex. Limnology and Oceanography, 26 (2), 201-218. doi:10.4319/lo.1981.26.2.0201

Gu, L., Qin, S., Lu, N., Zhao, Y., Zhou, Q., Zhang, L., . . . Yang, Z. (2020). Daphnia mitsukuri traits responding to predation cues alter its population dynamics. Ecological Indicators, 117, 106587. doi:10.1016/j.ecolind.2020.106587 
Gu, L., Qin, S., Zhu, S., Lu, N., Sun, Y., Zhang, L., . . . Yang, Z. (2020). Microcystis aeruginosa affects the inducible anti-predator responses of Ceriodaphnia cornuta .Environmental Pollution, 259 , 113952. doi:10.1016/j.envpol.2020.113952

Gu, L., Xu, X., Li, Y., Sun, Y., Zhang, L., Lyu, K., . . . Yang, Z. (2021). Induction and reversibility of Ceriodaphnia cornuta horns under varied intensity of predation risk and their defensive effectiveness against Chaoborus larvae. Freshwater Biology, 00 , 1- 11. doi:10.1111/fwb.13710

Hahn, M. A., Effertz, C., Bigler, L., \& von Elert, E. (2019). 5 alpha-cyprinol sulfate, a bile salt from fish, induces diel vertical migration in Daphnia . Elife, 8 , e44791. doi:10.7554/eLife.44791

Hales, N. R., Schield, D. R., Andrew, A. L., Card, D. C., Walsh, M. R., \& Castoe, T. A. (2017). Contrasting gene expression programs correspond with predator-induced phenotypic plasticity within and across generations in Daphnia . Molecular ecology, 26 (3), 5003-5015. doi:10.1111/mec.14213

Herzog, Q., \& Laforsch, C. (2013). Modality matters for the expression of inducible defenses: introducing a concept of predator modality.Bmc Biology, 11 , 113. doi:10.1186/1741-7007-11-113

Herzog, Q., Rabus, M., Ribeiro, B. W., \& Laforsch, C. (2016). Inducible defenses with a "Twist": Daphnia barbata abandons bilateral symmetry in response to an ancient predator. Plos One, 11 (2), e0148556. doi:10.1371/journal.pone.0148556

Heynen, M., Bunnefeld, N., \& Borcherding, J. (2017). Facing different predators: adaptiveness of behavioral and morphological traits under predation. current zoology, 63 (3), 249-257. doi:10.1093/cz/zow056

Kilham, S. S., Kreeger, D. A., Lynn, S. G., Goulden, C. E., \& Herrera, L. (1998). COMBO: a defined freshwater culture medium for algae and zooplankton. Hydrobiologia, 377 (1), 147-159. doi:10.1023/A:1003231628456

Kvile, K. O., Altin, D., Thommesen, L., \& Titelman, J. (2021). Predation risk alters life history strategies in an oceanic copepod.Ecology, 102 (1), e03214. doi:10.1002/ecy.3214

Laforsch, C., Ngwa, W., Grill, W., \& Tollrian, R. (2004). An acoustic microscopy technique reveals hidden morphological defenses inDaphnia . Proceedings of the National Academy of Sciences, 101 (45), 15911-15914. doi:10.1073/pnas.0404860101

Loose, C. J., \& Dawidowicz, P. (1994). Trade-offs in diel vertical migration by zooplankton: the costs of predator avoidance.Ecology, 75 (8), 2255-2263. doi:10.2307/1940881

Lurling, M. (2020). Grazing resistance in phytoplankton. Hydrobiologia, 848 , 237-249. doi:10.1007/s10750020-04370-3

Mahato, S., Morita, S., Tucker, A. E., Liang, X., Jackowska, M., Friedrich, M., . . . Zelhof, A. C. (2014). Common transcriptional mechanisms for visual photoreceptor cell differentiation among Pancrustaceans. PLOS Genetics, 10 (7), e1004484. doi:10.1371/journal.pgen.1004484

Miner, B. E., De Meester, L., Pfrender, M. E., Lampert, W., \& Hairston, N. G., Jr. (2012). Linking genes to communities and ecosystems:Daphnia as an ecogenomic model. Proceedings of the Royal Society B: Biological Sciences, 279 (1735), 1873-1882. doi:10.1098/rspb.2011.2404

Mitchell, M. D., Bairos-Novak, K. R., \& Ferrari, M. C. O. (2017). Mechanisms underlying the control of responses to predator odours in aquatic prey. Journal of Experimental Biology, 220 (11), 1937-1946. doi: $10.1242 /$ jeb.135137

Miyakawa, H., Imai, M., Sugimoto, N., Ishikawa, Y., Ishikawa, A., Ishigaki, H., . . . Cornette, R. (2010). Gene up-regulation in response to predator kairomones in the water flea, Daphnia pulex .BMC Developmental Biology, 10 (1), 45. doi:10.1186/1471-213x-10-45 
Mortazavi, A., Williams, B. A., McCue, K., Schaeffer, L., \& Wold, B. (2008). Mapping and quantifying mammalian transcriptomes by RNA-Seq.Nat Methods, 5 (7), 621-628. doi:10.1038/nmeth.1226

O'brien, W. (1987). Predation: direct indirect impacts on aquatic communities. In W. C. Kerfoot \& A. Sih (Eds.), Planktivory by freshwater fish: thrust and parry in the pelagia (pp. 3-16). Hanover, N.H.: University Press of New England.

Otte, K., Frohlich, T., Arnold, G., \& Laforsch, C. (2014). Proteomic analysis of Daphnia magna hints at molecular pathways involved in defensive plastic responses. BMC Genomics, 15 (1), 1-17. doi:10.1186/14712164-15-306

Pauwels, K., Stoks, R., \& De Meester, L. (2005). Coping with predator stress: interclonal differences in induction of heat-shock proteins in the water flea Daphnia magna. Journal of Evolutionary Biology, 18 (4), 867-872. doi:10.1111/j.1420-9101.2005.00890.x

Pauwels, K., Stoks, R., Decaestecker, E., \& De Meester, L. (2007). Evolution of heat shock protein expression in a natural population ofDaphnia magna . The American naturalist, 170 (5), 800-805. doi:10.1086/521956

Pietrzak, B., Pijanowska, J., \& Dawidowicz, P. (2017). The effect of temperature and kairomone on Daphnia escape ability: a simple bioassay. Hydrobiologia, 798 (1), 15-23. doi:10.1007/s10750-015-2539-z

Pijanowska, J., \& Kloc, M. (2004). Daphnia response to predation threat involves heat-shock proteins and the actin and tubulin cytoskeleton. Genesis, 38 (2), 81-86. doi:10.1002/gene.20000

Qin, S., Ma, L., Li, D., Huang, J., Zhang, L., Sun, Y., \& Yang, Z. (2021). Rising temperature accelerates the responses of inducible anti-predator morphological defenses of Ceriodaphnia cornuta but decreases the responsive intensity. Ecological Indicators, 120 , 106919. doi:10.1016/j.ecolind.2020.106919

Reede, T. (1995). Life history shifts in response to different levels of fish kairomones in Daphnia . Journal of Plankton Research, 17 (8), 1661-1667. doi:10.1093/plankt/17.8.1661

Riessen, H. P., \& Gilbert, J. J. (2019). Divergent developmental patterns of induced morphological defenses in rotifers andDaphnia : ecological and evolutionary context. Limnology and Oceanography, 64 (2), 541-557. doi:10.1002/lno.11058

Riessen, H. P., \& Trevett-Smith, J. B. (2009). Turning inducible defenses on and off: adaptive responses of Daphnia to a gape-limited predator. Ecology, 90 (12), 3455-3469. doi:10.2307/25660991

Ritschar, S., Rabus, M., \& Laforsch, C. (2020). Predator-specific inducible morphological defenses of a water flea against two freshwater predators. Journal of Morphology, 281 (6), 653-661. doi:10.1002/jmor.21131

Schwarzenberger, A., Courts, C., \& von Elert, E. (2009). Target gene approaches: Gene expression in Daphnia magna exposed to predator-borne kairomones or to microcystin-producing and microcystin-free Microcystis aeruginosa. BMC Genomics, 10 (1), 527. doi:10.1186/1471-2164-10-527

Scoville, A. G., \& Pfrender, M. E. (2010). Phenotypic plasticity facilitates recurrent rapid adaptation to introduced predators.Proceedings of the National Academy of Sciences, 107 (9), 4260-4263. doi:10.1073/pnas.0912748107

Selander, E., Kubanek, J., Hamberg, M., Andersson, M. X., Cervin, G., \& Pavia, H. (2015). Predator lipids induce paralytic shellfish toxins in bloom-forming algae. Proceedings of the National Academy of Sciences, 112 (20), 6395-6400. doi:10.1073/pnas.1420154112

Steiner, U. K., \& Auld, J. R. (2012). Why is the jack of all trades a master of none? Studying the evolution of inducible defences in aquatic systems. In C. Bronmark \& L. A. Hansson (Eds.), Chemical Ecology in Aquatic Systems (pp. 172). New York, NY: Oxford University Press.

Stibor, H. (2002). The role of yolk protein dynamics and predator kairomones for the life history of Daphnia magna . Ecology, 83 (2), 362-369. doi:10.2307/2680020 
Stibor, H., \& Luning, J. (1994). Predator-induced phenotypic variation in the pattern of growth and reproduction in Daphnia hyalina(Crustacea: Cladocera). Functional Ecology, 8 , 97-101. doi:10.2307/2390117

Stibor, H., \& Navarra, D. M. (2000). Constraints on the plasticity ofDaphnia magna influenced by fishkairomones. Functional Ecology, 14 (4), 455-459. doi:10.1046/j.1365-2435.2000.00441.x

Stoks, R., Govaert, L., Pauwels, K., Jansen, B., \& De Meester, L. (2016). Resurrecting complexity: the interplay of plasticity and rapid evolution in the multiple trait response to strong changes in predation pressure in the water flea Daphnia magna . Ecology letters, 19 (2), 180-190. doi:10.1111/ele.12551

Swift, M. C. (1992). Prey capture by the four larval instars ofChaoborus crystallinus . Limnology and Oceanography, 37 (1), 14-24. doi:10.4319/lo.1992.37.1.0014

Tollrian, R. (1993). Neckteeth formation in Daphnia pulex as an example of continuous phenotypic plasticity: morphological effects ofChaoborus kairomone concentration and their quantification.Journal of Plankton Research, 15 (11), 1309-1318. doi:10.1093/plankt/15.11.1309

Tollrian, R. (1995). Predator-induced morphological defenses: Costs, life history shifts, and maternal effects in Daphnia pulex .Ecology, 76 (6), 1691-1705. doi:10.2307/1940703

Tollrian, R., \& Harvell, C. D. (1999). The ecology and evolution of inducible defenses . Princeton, NJ: Princeton University Press.

Von Elert, E., Agrawal, M. K., Gebauer, C., Jaensch, H., Bauer, U., \& Zitt, A. (2004). Protease activity in gut of Daphnia magna : evidence for trypsin and chymotrypsin enzymes. Comparative Biochemistry and Physiology Part B: Biochemistry and Molecular Biology, 137 (3), 287-296. doi:10.1016/j.cbpc.2003.11.008

Weiss, L. C., Albada, B., Becker, S. M., Meckelmann, S. W., Klein, J., Meyer, M., . . . Tollrian, R. (2018). Identification of Chaoboruskairomone chemicals that induce defences in Daphnia . Nature Chemical Biology, 14 (12), 1133-1139. doi:10.1038/s41589-018-0164-7

Weiss, L. C., Leese, F., Laforsch, C., \& Tollrian, R. (2015). Dopamine is a key regulator in the signalling pathway underlying predator-induced defences in Daphnia. Proceedings of the Royal Society B: Biological Sciences, 282 (1816), 20151440. doi:10.1098/rspb.2015.1440

Westra, Edze R., van Houte, S., Oyesiku-Blakemore, S., Makin, B., Broniewski, Jenny M., Best, A., . . . Buckling, A. (2015). Parasite exposure drives selective evolution of constitutive versus inducible defense. Current Biology, 25 (8), 1043-1049. doi:10.1016/j.cub.2015.01.065

Yin, M., Laforsch, C., Lohr, J. N., \& Wolinska, J. (2011). Predator-induced defense makes Daphnia more vulnerable to parasites. Evolution, 65 (5), 1482-1488. doi:10.1111/j.1558-5646.2011.01240.x

Zhang, C., Jones, M., Govaert, L., Viant, M., De Meester, L., \& Stoks, R. (2021). Resurrecting the metabolome: Rapid evolution magnifies the metabolomic plasticity to predation in a natural Daphniapopulation. Molecular ecology, 30 , 2285-2297. doi:10.1111/mec.15886

Zhou, X., Liao, W., Liao, J., Liao, P., \& Lu, H. (2015). Ribosomal proteins: functions beyond the ribosome. Journal of Molecular Cell Biology, 7 (2), 92-104. doi:10.1093/jmcb/mjv014

\section{Data Accessibility}

Sequence data is uploaded to the Sequence Read Archive (SRA) with accession PRJNA735795.

\section{Author contributions}

LG, SA, LZ, and ZY designed the experiment. LG, SQ, YS, and JH performed the experiment and analyzed the data. LG and ZY wrote the first draft of the manuscript. All authors participated in discussions and editing of the manuscript. 


\section{Tables and Figures}

Tables

Table 1. Results of the MANOVA, Two-way ANOVA, and Scheirer-Ray-Hare on different traits of C. cornuta . Significant results $(P<0.05)$ are given in bold.

\begin{tabular}{|c|c|c|c|c|}
\hline MANOVA & MANOVA & Chaoborus larvae $(\mathrm{CH})$ & Chaoborus larvae $(\mathrm{CH})$ & Chaoborus larvae (C \\
\hline & & $F$ & $d f$ & Pillai \\
\hline All traits & All traits & 26.615 & 9 & 0.952 \\
\hline Two-way ANOVA & Two-way ANOVA & $F$ & $d f$ & $P$ \\
\hline Size at maturity & Size at maturity & 3.811 & 1,35 & 0.059 \\
\hline Size at 16 th day & Size at 16 th day & 4586 & 1,34 & $<0.001$ \\
\hline Neonate size & Neonate size & 0.368 & 1,20 & 0.551 \\
\hline Time to first brood & Time to first brood & 1.041 & 1,35 & 0.315 \\
\hline Total offspring number & Total offspring number & 15.158 & 1,34 & $<0.001$ \\
\hline Brood number & Brood number & 2.269 & 1,34 & 0.141 \\
\hline Average brood size & Average brood size & 10.626 & 1,34 & 0.003 \\
\hline Scheirer-Ray-Hare & Scheirer-Ray-Hare & $H$ & $d f$ & $P$ \\
\hline Horns at maturity & Horns at maturity & 24.743 & 1,34 & $<0.001$ \\
\hline Horns at 16 th day & Horns at 16 th day & 12.46 & 1,34 & $<0.001$ \\
\hline
\end{tabular}

Table 2. Key genes of significant function pathways in response to fish and Chaoborus larvae predation risks.

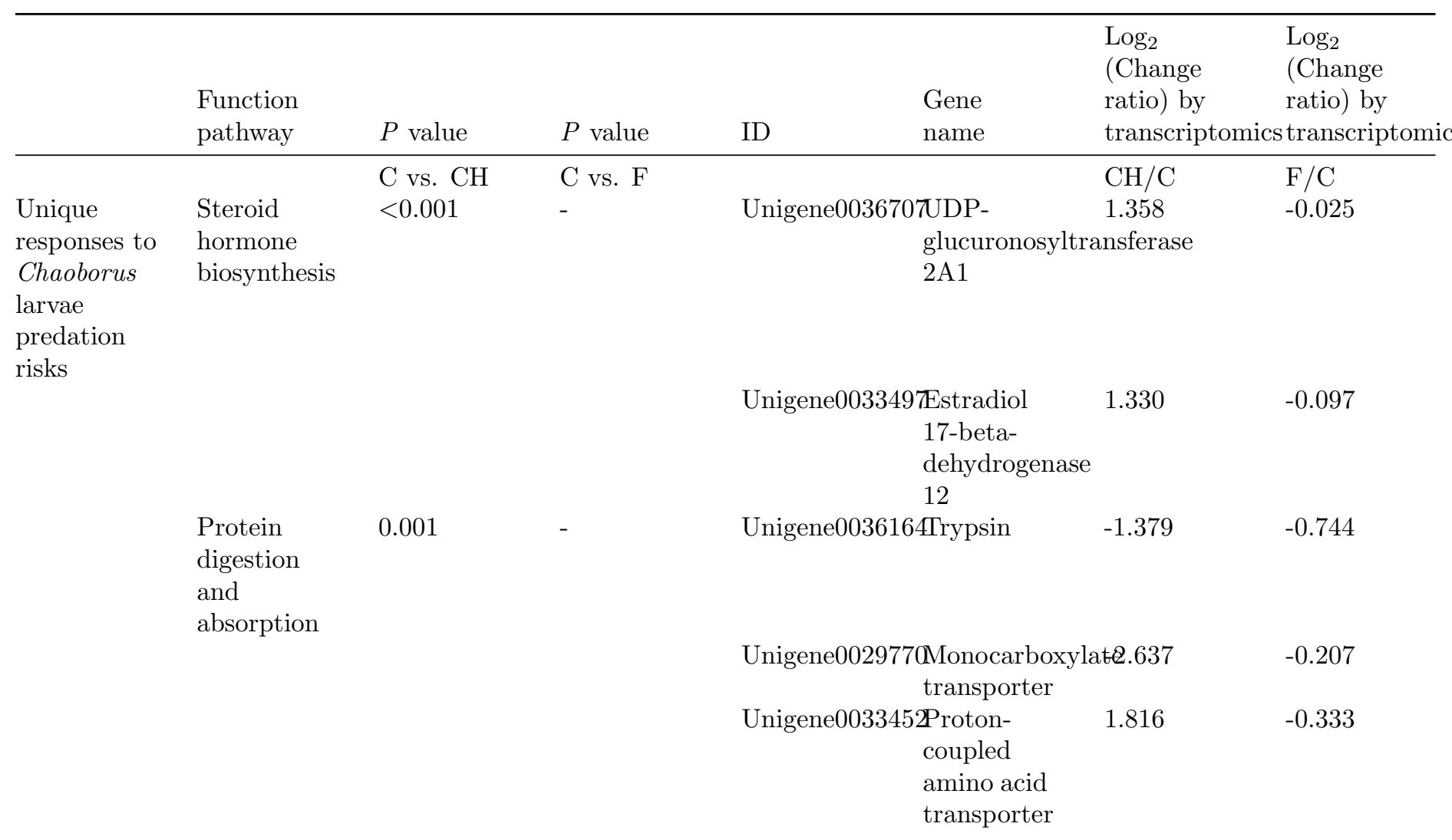




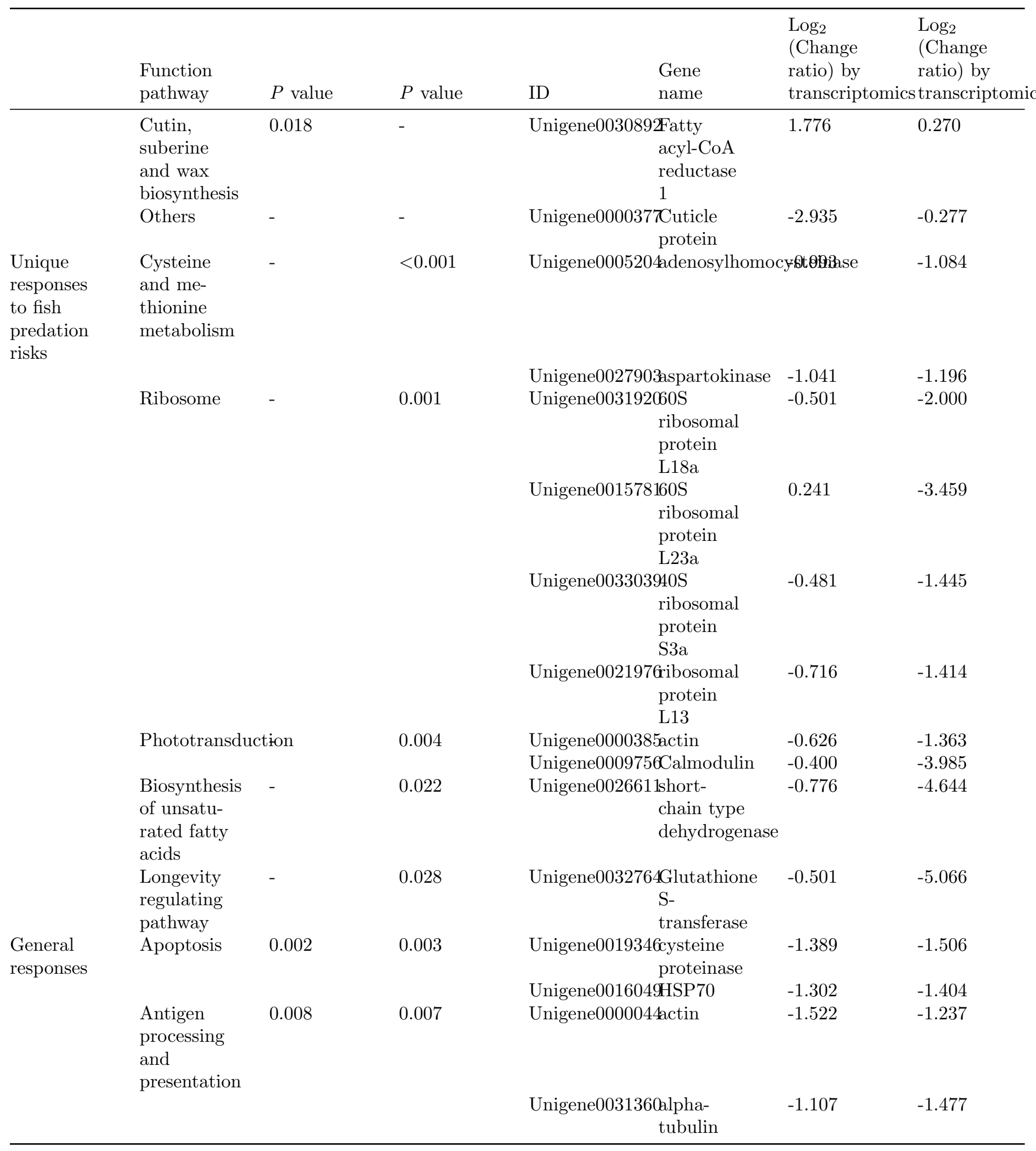




\section{Figures}

\section{Hosted file}

image1.emf available at https://authorea.com/users/424411/articles/529504-inducibleresponses-to-antagonistic-predation-risks-are-not-in-a-dilemma-evidences-from-multitraits-and-transcriptome-of-ceriodaphnia

Figure 1 . Morphology and life history responses of C. cornuta under fish and Chaoborus larvae predation risks. The dark line with asterisks indicates significant differences between treatments with and without fish predation risks $\left({ }^{*} P<0.05,{ }^{* *} P<0.01\right.$ and $\left.{ }^{* * *} P<0.001\right)$. The upper and lower case letters indicate differences among treatments of absent and present Chaoborus larvae predation risks, respectively. The error bar indicates the standard error.

\section{Hosted file}

image2.emf available at https://authorea.com/users/424411/articles/529504-inducibleresponses-to-antagonistic-predation-risks-are-not-in-a-dilemma-evidences-from-multitraits-and-transcriptome-of-ceriodaphnia

Figure 2 . Correlation analysis (A) and principal component analysis (B) of gene expression of $C$. cornuta under different predation risks. (C) Correlation between RNA-Seq and RT-qPCR data.

\section{Hosted file}

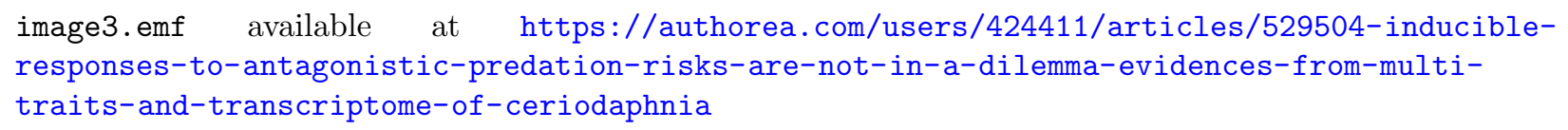

Figure 3 . Quantitative analysis of differentially expressed genes of $C$. cornuta in different treatments.

\section{Hosted file}

image4.emf available at https://authorea.com/users/424411/articles/529504-inducibleresponses-to-antagonistic-predation-risks-are-not-in-a-dilemma-evidences-from-multitraits-and-transcriptome-of-ceriodaphnia

Figure 4. Correlation relationships within different responses in individual traits and transcriptional expressions. The asterisks indicate significant Pearson correlations between two traits or genes $(P<0.05)$. The correlations between unique responses to different predators are in squares.

\section{Hosted file}

image5.emf available at https://authorea.com/users/424411/articles/529504-inducibleresponses-to-antagonistic-predation-risks-are-not-in-a-dilemma-evidences-from-multitraits-and-transcriptome-of-ceriodaphnia

Figure 5. Diagram of pathways of $C$. cornuta in response to fish and Chaoborus larvae predation risks. 